\title{
Frugivoria em morcegos (Mammalia, Chiroptera) no Parque Estadual Intervales, sudeste do Brasil ${ }^{1}$
}

\author{
Fernando C. Passos ${ }^{2}$, Wesley R. Silva ${ }^{3}$, Wagner A. Pedro ${ }^{4} \&$ Marcela R. Bonin ${ }^{2,5}$
}

\author{
1 Contribuição número 1401 do Departamento de Zoologia, Universidade Federal do Paraná. \\ ${ }^{2}$ Departamento de Zoologia, Universidade Federal do Paraná. Caixa Postal 19020, 81531-980 Curitiba, Paraná, Brasil. \\ E-mail: fpassos@ufpr.br \\ ${ }^{3}$ Departamento de Zoologia, Universidade Estadual de Campinas. Caixa Postal 6109, 13083-970 Campinas, São Paulo, \\ Brasil. \\ ${ }^{4}$ Departamento de Apoio, Produção e Saúde Animal, Universidade Estadual Paulista. Caixa Postal 341, 16050-680 \\ Araçatuba, São Paulo, Brasil. \\ ${ }^{5}$ Bolsista PIBIC/CNPq.
}

\begin{abstract}
Frugivory in bats (Mammalia, Chiroptera) at the Intervales State Park, Southeastern Brazil. This study was carried out at the Intervales State Park, an Atlantic Rain Forest area in Southeastern Brazil. Bats were monthly mist netted over a full year, and fecal samples were collected for dietary analysis. The seeds found in each sample were identified in the laboratory under a stereoscopic microscope by comparison with seeds taken from ripe fruits collected in the study area. Three hundred and seventy one bats were collected, of which 316 $\mathbf{( 8 5 . 2 \% )}$ were frugivorous. The total number of fecal samples with seeds and/or pulp was 121. Sturnira lilium (E. Geoffroy, 1810) was the most abundant species in the study area ( $\mathrm{n}=157$ captures) and Solanaceae fruits accounted for $78.5 \%$ of the fecal samples with seeds $(\mathrm{n}=56)$. Artibeus fimbriatus Gray, $1838(\mathrm{n}=21$ samples) fed mostly on Cecropiaceae (38\%) and Moraceae fruits (24\%), and Artibeus lituratus (Olfers, 1818) ( $\mathrm{n}=7$ samples) on Cecropiaceae (57\%) and Moraceae (29\%). Carollia perspicillata (Linnaeus, 1758) ( $\mathrm{n}=16$ samples) fed mostly on Piperaceae fruits $(56,3 \%)$, but Solanaceae $(31,3 \%)$ and Rosaceae seeds $(12,5 \%)$ were also found in feces. Overall, seeds found in bat feces belong to eight plant families: Solanaceae ( $n=67$ samples); Cecropiaceae $(n=14)$; Piperaceae ( $n=14)$; Moraceae $(n=8)$; Rosaceae $(n=3)$; Cucurbitaceae $(n=3)$; Cluseaceae $(n=1)$, and Araceae $(n=1)$. The close association of different bat species with fruits of certain plant families and genus may be related to a possible mechanism of resource partitioning that shapes the structure of the community.
\end{abstract}

KEY WORDS. Artibeus, Carollia, diet, Sturnira.

A importância ecológica dos morcegos (Ordem Chiroptera), sua alta diversidade e sua abundância nas regiões tropicais (CoLE $\&$ WiLSON 1996, NoWAC 1991) tornam este grupo um interessante objeto de estudos. Trabalhos em sistemática, fisiologia, distribuição geográfica, levantamento de espécies e ecologia vêm sendo desenvolvidos em diversas localidades, contribuindo assim para o melhor conhecimento desses mamíferos (Bernard 2002, Charles-Dominique 1986, Emmons \& Feer 1997, Heithaus et al. 1975, Lim et al. 2003). A ecologia alimentar de morcegos provê informações extremamente úteis para o entendimento dos mecanismos de partilha de recursos que regulam as relações tróficas, e que são responsáveis pela alta diversidade deste grupo nas regiões tropicais (Heithaus et al. 1975,
Marinho-Filho 1991, Muller \& Reis 1992, Pedro \& Taddei 1997, Terborgh 1986, Willig et al. 1993).

Morcegos frugívoros formam uma parcela considerável das comunidades de morcegos em ambientes neotropicais (EMmONs \& FeEr 1997), havendo para o Brasil informações específicas sobre a dieta frugívora de algumas espécies (e.g. GaLETTI \& Morellato 1994, Marinho-Filho 1991, Muller \& Reis 1992; Pedro \& Passos 1995, Pedro \& Taddei 1997, Reis \& Guillaumet 1983, Uieda \& Vasconcellos-Neto 1985, Willig et al. 1993, ZorTéa \& ChIARello 1994). Apesar destes trabalhos, ainda existem poucas informações sobre a ecologia alimentar das espécies de morcegos frugívoros no Brasil, especialmente no domínio da Mata Atlântica do sudeste, uma das regiões florestais mais 
ameaçadas do globo (Fonseca 1985, Fonseca et al. 1994, Terborgh 1992). A dispersão de sementes por morcegos frugívoros contribui para o estabelecimento de muitas espécies de plantas pioneiras, auxiliando os mecanismos de regeneração e sucessão secundária em áreas tropicais (CHARLEs-DominIQue 1986, GoRCHOv et al. 1993).

São apresentadas informações sobre a dieta frugívora e a abundância de espécies de morcegos frugívoros de uma área de Mata Atlântica, discutindo as possíveis preferências alimentares e partilha de recursos entre as espécies estudadas.

\section{MATERIAL E MÉTODOS}

$O$ estudo foi realizado no Parque Estadual de Intervales, município de Ribeirão Grande/SP $\left(24^{\circ} 16^{\prime} \mathrm{S}, 48^{\circ} 24^{\prime} \mathrm{W}\right)$, numa área próxima à sede administrativa do parque. A região apresenta vegetação de Mata Atlântica e não existe uma estação seca bem definida, embora a precipitação seja mais baixa entre maio e setembro (Trajano 1996). As chuvas anuais variam de 1000 a $2000 \mathrm{~mm}$ e a média de temperatura anual é cerca de $20^{\circ} \mathrm{C}$ (Fenton et al. 1999). Entre os anos de 1994 a 1996 a precipitação anual variou de 1579 a $1934 \mathrm{~mm}$, com variações mensais de 20 a $470 \mathrm{~mm}$, e as temperaturas de 10 a $24^{\circ} \mathrm{C}$ (Dados fornecidos pelo DAEE de São Paulo).

Coletas mensais foram realizadas ao longo de treze meses, de janeiro de 1999 a janeiro de 2000, através de capturas com redes de neblina. Durante esse período as capturas de morcegos ocorreram de dois até 10 dias por mês. Ao longo dos meses, entre seis e 12 redes foram dispostas diariamente por aproximadamente seis horas, desde o início do anoitecer. Os morcegos capturados foram identificados, medidos, pesados, colocados em sacos de algodão para defecar e posteriormente liberados. Alguns exemplares foram sacrificados para servir como material testemunho ou mesmo para a identificação precisa da espécie (Tab. I). As fezes coletadas foram então acondicionadas e etiquetadas.

No laboratório essas amostras foram observadas sob lupa para a identificação do material fecal. As sementes encontradas foram lavadas e identificadas em nível de família, gênero ou espécie através da comparação com sementes de uma coleção

Tabela I. Espécies tombadas em coleções como material testemunho.

\begin{tabular}{|c|c|c|}
\hline Espécie & Coleção & Número \\
\hline Peropteryx macrotis (Wagner, 1843) & L. Chiroptera ${ }^{1}$ & FP114, FP115, FP116 e FP117 \\
\hline \multirow[t]{2}{*}{ Furipterus horrens (F. Cuvieer, 1828) } & $\mathrm{MHNCI}^{2}$ & $?^{3}$ \\
\hline & L. Chiroptera & FP254 \\
\hline Anoura caudifera (E. Geoffroy, 1818) & L. Chiroptera & FP277 \\
\hline Anoura geoffroyi Gray, 1838 & UFPR & FP124 \\
\hline Artibeus fimbriatus Gray, 1838 & L. Chiroptera & FP151, FP158, FP159, FP317, FP320, FP321, FP325, FP333 \\
\hline Artibeus jamaicensis Leach, 1821 & L. Chiroptera & FP104, FP329 \\
\hline Artibeus lituratus (Olfers, 1818) & L. Chiroptera & FP109, FP118, FP161, FP319, FP342, FP363 \\
\hline Artibeus obscurus Schinz, 1821 & L. Chiroptera & FP123 \\
\hline Carollia perspicillata (Linnaeus, 1758) & L. Chiroptera & FP23, FP187 \\
\hline Chiroderma doriae Thomas, 1891 & L. Chiroptera & FP343 \\
\hline Chrotopterus auritus (Peters, 1856) & UFPR & FP99 \\
\hline Desmodus rotundus (E. Geoffroy, 1810) & $\mathrm{UFPR}^{4}$ & FP150 \\
\hline Lonchorhina aurita Thomes, 1863 & L. Chiroptera & FP31 \\
\hline Pygoderma bilabiatum (Wagner, 1843) & L. Chiroptera & FP176 \\
\hline Platyrrhinus recifinus (Thomas, 1901) & L. Chiroptera & FP10 \\
\hline Sturnira lilium (E. Geoffroy, 1810) & UFPR & FP128, FP129, FP130 \\
\hline Sturnira tildae De la Torre, 1959 & L. Chiroptera & FP 237, FP238, FP299 \\
\hline Histiotus velatus (I. Geoffroy, 1824) & UFPR & FP146 \\
\hline Myotis nigricans (Schunz, 1821) & L. Chiroptera & FP108, FP201 \\
\hline Myotis ruber (E. Geoffroy, 1806) & L. Chiroptera & FP30 \\
\hline Myotis indeterminados & L. Chiroptera & FP196, FP197, FP228 \\
\hline
\end{tabular}

(1) Laboratório de Chiroptera, Departamento de Apoio, Produção e Saúde Animal, UNESP, Araçatuba; (2) Museu de História Natural do Capão da Imbuia, Curitiba; (3) Indeterminado; (4) Coleção de Mastozoologia do Departamento de Zoologia da UFPR, Curitiba.

Revista Brasileira de Zoologia 20 (3): 511-517, setembro 2003 
de referência montada a partir de frutos coletados na área. As plantas cujos frutos foram coletados foram marcadas e as exsicatas foram enviadas a especialistas para a identificação. Os morcegos frugívoros foram considerados aqueles que apresentaram um dieta constituída basicamente de frutos. As amostras das fezes que continham uma única espécie de semente foram consideradas como uma única amostra, enquanto aquelas que apresentaram duas ou mais sementes de espécies diferentes foram consideradas como duas ou mais amostras. Assim, o número de amostras para o cálculo da dieta foi considerado o total das amostras produzidas por cada espécie de morcego. É importante ressaltar que esta técnica de análise de fezes, embora de uso comum em estudos sobre alimentação de morcegos frugívoros (Bernard 2002, Pedro \& Taddei 1997, Sipinski \& Reis 1995, entre outros), é limitada para caracterizar a totalidade da dieta desses animais, uma vez que muitos frutos com sementes grandes que não atravessam o tubo digestivo podem também ser consumidos (Galetti \& Morellato 1994, Zortéa \& Chiarello 1994).

\section{RESULTADOS}

Durante o período de estudo foram capturados 371 morcegos, sendo que $316(85,2 \%)$ pertenciam a espécies frugívoras (Tab. II). Os frugívoros mais abundantes na área de estudo foram: Sturnira lilium (157 capturas), Artibeus fimbriatus (74), Artibeus lituratus (29) e Carollia perspicillata (24). Para estas espécies, o percentual de amostras fecais com sementes revelou que Cecropiaceae, Moraceae, Piperaceae e Solanaceae são algumas das famílias de plantas mais consumidas pelos morcegos frugívoros na área de estudo (Fig. 1).

A dieta frugívora de Sturnira lilium foi a mais diversificada com oito famílias vegetais sendo consumidas, porém concentrando-se em frutos de Solanaceae $(78,5 \%)$, principalmente do gênero Solanum (Fig. 1). Artibeus fimbriatus apresentou principalmente sementes de Cecropiaceae (38\%) e Moraceae (24\%) em suas fezes, e em menor quantidade sementes de Solanaceae, Piperaceae e Cucurbitaceae. Nas poucas amostras fecais de Artibeus lituratus predominaram sementes de Cecropiaceae (57\%), além de Moraceae (29\%) e Solanaceae (14\%). Carollia perspicillata também concentrou sua dieta em poucas famílias, sendo encontradas somente sementes de Piperaceae (56\%), Solanaceae (31\%) e Rosaceae $(12,5 \%)$.

As espécies Artibeus jamaicensis, Pygoderma bilabiatum e Sturnira tildae apresentaram poucas fezes com sementes ou com a presença somente de polpa não identificável. Platyrrhinus recifinus, Artibeus obscurus e Chiroderma dorie não defecaram.

Ao todo foram obtidas 121 amostras fecais das sete espécies de morcegos frugívoros em Intervales, das quais 106 continham sementes e 15 somente restos de polpa não identificável (Tab. III). As plantas consumidas pelos morcegos representam oito famílias e 22 espécies identificadas pelo menos em gênero, sendo que as sementes de Solanaceae, Cecropiaceae e Piperaceae ocorreram em $78,5 \%$ das amostras (Tab. III).

\section{DISCUSSÃO}

Estudos realizados com morcegos no Parque Estadual Intervales abordaram composição da fauna, comparação entre diferentes técnicas de amostragem da comunidade, além de informações sobre a dieta e o comportamento de forrageio de morcegos insetívoros (Fenton et al. 1999, PortFors et al. 2000). Entretanto, esta é a primeira análise mais abrangente sobre a
Tabela II. Espécies de morcegos capturadas no Parque Estadual Intervales e suas respectivas guildas alimentares e freqüência de captura.

\begin{tabular}{|c|c|c|c|}
\hline Familía & Espécie & Guilda & Capturas \\
\hline Emballonuridae & Peropteryx macrotis & Insetívoro & 6 \\
\hline \multirow[t]{16}{*}{ Phyllostomidae } & Chrotopterus auritus & Carnívoro & 3 \\
\hline & Lonchorhina aurita & Insetívoro & 15 \\
\hline & Micronycteris microtis & Insetívoro & 1 \\
\hline & Anoura caudifer & Nectarívoro & 3 \\
\hline & Anoura geoffroyi & Nectarívoro & 2 \\
\hline & Carollia perspicillata & Frugívoro & 24 \\
\hline & Artibeus fimbriatus & Frugívoro & 74 \\
\hline & Artibeus jamaicensis & Frugívoro & 5 \\
\hline & Artibeus lituratus & Frugívoro & 29 \\
\hline & Artibeus obscurus & Frugívoro & 1 \\
\hline & Chiroderma doriae & Frugívoro & 1 \\
\hline & Platyrrhinus recifinus & Frugívoro & 1 \\
\hline & Pygoderma bilabiatum & Frugívoro & 10 \\
\hline & Sturnira lilium & Frugívoro & 157 \\
\hline & Sturnira tildae & Frugívoro & 14 \\
\hline & Desmodus rotundus & Hematófago & 9 \\
\hline Furipteridae & Furipterus horrens & Insetívoro & 6 \\
\hline \multirow[t]{4}{*}{ Vespertilionidae } & Histiotus velatus & Insetívoro & 1 \\
\hline & Myotis nigricans & Insetívoro & 1 \\
\hline & Myotis ruber & Insetívoro & 1 \\
\hline & Myotis indeterminado & Insetívoro & 4 \\
\hline Total (4 famílias) & 24 espécies & 5 guildas & 371 \\
\hline
\end{tabular}

dieta de morcegos frugívoros para aquela Unidade de Conservação.

A abundância de morcegos frugívoros encontrados neste estudo (Tab. II) reflete a importância desta guilda nas comunidades de morcegos de Mata Atlântica, onde muitas espécies de plantas quiropterocóricas são capazes de manter uma comunidade diversificada de morcegos ao longo de todo o ano. Pedro et al. (2001) ressaltam a importância das principais espécies de morcegos frugívoros (A. lituratus, $C$. perspicillata e $S$. lilium) na dinâmica da comunidade. Além disso, essa abundância de frugívoros pode refletir também a seletividade do método de coleta por rede de neblina, uma vez espécies insetívoras como os exemplares das famílias Vespertilionidae e Molossidae podem evitar essas redes (ARIta 1993, Pedro \& TAdDei 1997).

A dominância de Sturnira lilium já foi relatada em outros estudos de comunidades de morcegos no Brasil (MARINHO-FILHO 1991, Pedro \& TAdDei 1997). Em algumas localidades Artibeus Leach, 1821 pode ser dominante (Muller \& ReIs 1992, Reis et al. 2000, Pedro et al. 2001, Rui \& Fabián 1997, SiPInSKi \& Reis 1995)

Revista Brasileira de Zoologia 20 (3): 511-517, setembro 2003 
Tabela III. Freqüência de ocorrência de sementes nas amostras fecais de sete espécies de morcegos frugívoros do Parque Estadual Intervales, Ribeirão Grande, São Paulo. (AF) Artibeus fimbriatus, (AL) A. lituratus, (AJ) A. jamaicensis, (CP) Carollia perspicillata, (PB) Pygoderma bilabiatum, (SL) Sturnira lilium, (ST) Sturnira tildae.

\begin{tabular}{|c|c|c|c|c|c|c|c|c|}
\hline \multirow{2}{*}{$\begin{array}{c}\text { Plantas } \\
\text { (Famílias/Espécies) }\end{array}$} & \multicolumn{8}{|c|}{ Espécies de morcegos } \\
\hline & $\mathrm{AF}$ & $\mathrm{AL}$ & AJ & $\mathrm{CP}$ & $\mathrm{PB}$ & SL & ST & Total \\
\hline \multicolumn{9}{|l|}{ Araceae } \\
\hline Philodendron apendiculatum Nadruz \& Mayo & & & & & & 1 & & 1 \\
\hline \multicolumn{9}{|l|}{ Cecropiaceae } \\
\hline Cecropia glaziovi Snethlage & 5 & 3 & 1 & & & 1 & & 10 \\
\hline Cecropia pachystachia Trec. & 3 & 1 & & & & & & 4 \\
\hline Cucurbitaceae & 1 & & & & & 2 & & 3 \\
\hline \multicolumn{9}{|l|}{ Cluseaceae } \\
\hline Vismia sp. Vand. & & & & & & 1 & & 1 \\
\hline \multicolumn{9}{|l|}{ Moraceae } \\
\hline Fícus luschnatiana (Miq.) Miq. & 2 & 1 & & & & & & 3 \\
\hline Ficus sp. L. & 3 & 1 & & & & 1 & & 5 \\
\hline \multicolumn{9}{|l|}{ Piperaceae } \\
\hline Piper aduncum Linnaeus & & & & 2 & & & & 2 \\
\hline Piper dilatatum L.C. Rich. & & & & 5 & & & & 5 \\
\hline Piper sp. L. & 1 & & & 2 & & 4 & & 7 \\
\hline \multicolumn{9}{|l|}{ Rosaceae } \\
\hline Rubus brasiliensis Mart. & & & & 2 & & 1 & & 3 \\
\hline \multicolumn{9}{|l|}{ Solanaceae } \\
\hline Aureliana sp. Sendt. & & & & & & 1 & & 1 \\
\hline Solanum cinnamomeum Sendt. & & & & & & 1 & & 1 \\
\hline Solanum megalochiton Mart. & & & & 1 & & & & 1 \\
\hline Solanum cf. paranaense Dunal & & & & 2 & & 2 & 1 & 5 \\
\hline Solanum rufescens Sendt. & & & & & & 1 & & 1 \\
\hline Solanum santaecatharinae Dunal & & & & & & 3 & & 3 \\
\hline Solanum scuticum M. Nee & & 1 & & & & 1 & 1 & 3 \\
\hline Solanum subsylvestris L.B. Smith \& Dawns & & & & & & 3 & & 3 \\
\hline Solanum swartzianum Roem. \& Schult & & & & & & 3 & & 3 \\
\hline Solanum variabile Mart. & & & & 1 & & 3 & & 4 \\
\hline Solanum sp. L. & 1 & & & 1 & & 15 & 3 & 20 \\
\hline Vassobia breviflora (Sendt.) Hunz & 1 & & & & & 11 & & 12 \\
\hline \multicolumn{9}{|l|}{ Famílias Indeterminadas } \\
\hline Indeterminadas & 4 & & & & & 1 & & 5 \\
\hline Polpa sem sementes & 4 & & & 2 & 1 & 8 & & 15 \\
\hline Total & 25 & 7 & 1 & 18 & 1 & 64 & 5 & 121 \\
\hline
\end{tabular}

mesmo co-ocorrendo com Sturnira lilium. Em outras localidades Carollia perspicillata se apresenta como a espécie dominante (Bernard 2002, Pedro \& Passos 1995, Pedro \& Taddei 2002, STONER 2001). No Parque Estadual Intervales, Sturnira lilium foi mais abundante que as quatro espécies de Artibeus juntas. Entretanto, tal fato pode ser explicado pela ausência de capturas de morcegos do gênero Artibeus durante os meses mais frios, período que coincide com a ausência de sua principal fonte de alimento na região (frutos de Cecropiaceae), indicando que estas espécies poderiam abandonar a área em busca de locais com maior disponibilidade de alimento. Tal fato, de possibilidade de migração relacionada a disponibilidade de alimento,

Revista Brasileira de Zoologia 20 (3): 511-517, setembro 2003 
Artibeus fimbriatus ( $\mathrm{n}=21$ amostras)

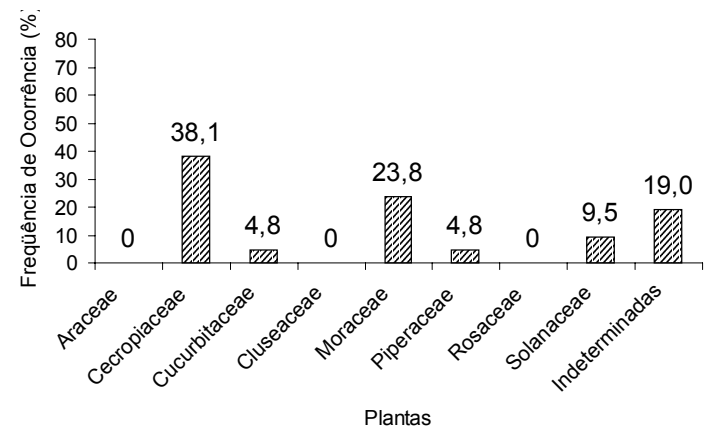

Artibeus lituratus ( $\mathrm{n}=7$ amostras)

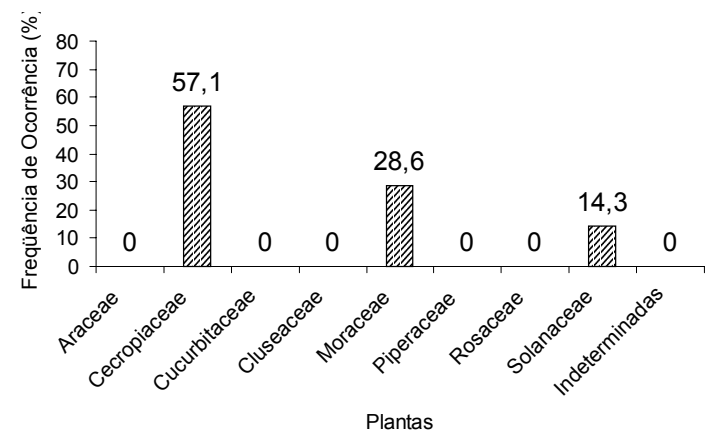

Carollia perspicillata $(\mathrm{n}=16$ amostras)

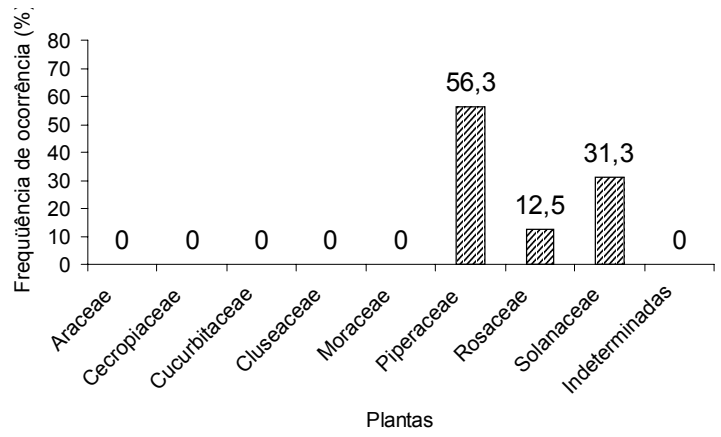

Sturnira lilium ( $\mathrm{n}=56$ amostras)

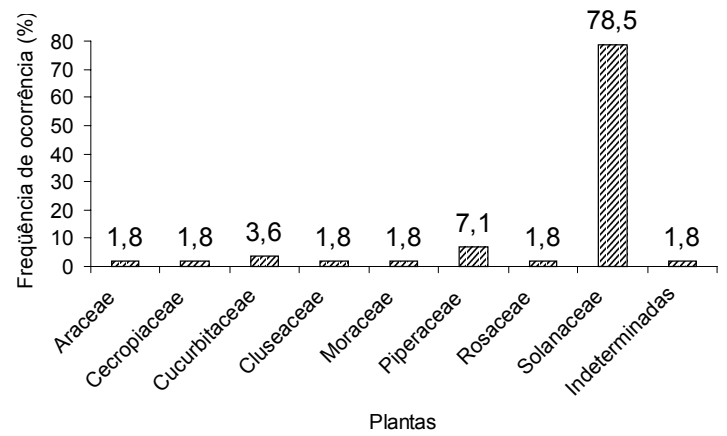

Figura 1. Freqüência de ocorrência (\%) de diferentes famílias vegetais nas amostras fecais com sementes encontradas em Artibeus fimbriatus, A. lituratus, Carollia perspicillata e Sturnira lilium no Parque Estadual de Intervales.

foi também comentada por Pedro \& Taddei (2002) para Platyrrhinus lineatus (E. Geoffroy, 1810), e deve ser cuidadosamente averiguado.

Além disso, a comunidade de morcegos frugívoros parece estar adaptada à partilha de recursos alimentares (FLEMING 1986, Muller \& Reis 1992, Marinho-Filho 1991), pois, pelo menos em relação às quatro espécies mais abundantes, é possível detectar uma tendência a dietas especializadas em determinadas famílias de plantas, como acontece entre Sturnira spp. e Solanaceae, Artibeus spp. e Cecropiacae/Moraceae, Carollia perspicillata e Piperaceae (Fig. 1). Plantas destas famílias são abundantes no mosaico de ambientes que constituem a Mata Atlântica, mas principalmente em habitats mais abertos como as bordas de mata, clareiras e ao longo de caminhos e trilhas, ambientes geralmente freqüentados por morcegos frugívoros. De maneira geral, a distribuição dos recursos e sua abundância são importantes fatores determinando os padrões de forrageio das espécies de morcegos (Heithaus et al. 1975).

A preferência de Sturnira lilium por frutos de Solanum e de Carollia perspicillata por Piper já tem sido observada em outros estudos e parece estar relacionada a mecanismos de partição de recursos permitindo a coexistência dessas espécies (Fleming 1986, Heithaus et al. 1975, Marinho-Filho 1991, Мikich 2002, Muller \& Reis 1992, Pedro \& Passos 1995, Pedro \& Taddei 1997, Sipinski \& Reis 1995). As espécies de Artibeus são conhecidas na literatura pelo grande consumo de Ficus e Cecropia (Fleming 1986, Gardner 1977, Zortéa \& Chiarello 1994). Na área de estudo, porém, uma espécie muito abundante de figueira é Ficus luschnatiana, que apresenta frutos aparentemente de baixa qualidade, pouco suculentos e de maior rigidez, quando comparados às outras espécies de figueiras. Isto poderia explicar não somente a falta de dominância de Artibeus spp. naquela região como também a raridade de outras espécies conhecidas pelo alto consumo de Ficus, como Plathyrrhinus recifinus (Muller \& Reis 1992) e Vampyressa pusilla (Wagner, 1843) (Pedro et al. 1997) (Tab. II).

A ausência de sementes nas fezes de Pygoderma bilabiatum (Tab. III) pode indicar que indivíduos desta espécie podem preferir o consumo de polpa e evitar engolir as sementes. Talvez eles pudessem ter sido capturados antes de se alimentar, mas como os exemplares desta espécie foram capturados em média às 23:30 h, é de se esperar que os indivíduos nesse horário já tenham se alimentado. São necessárias maiores informações sobre a dieta frugívora desta espécie, assim como para as outras espécies cujas amostras de fezes não permitiram uma análise mais aprofundada sobre sua dieta (A. jamaicensis, $A$. obscurus, $S$. tildae, $P$. recifinus e $C$. dorie).

A identificação das espécies de Solanaceae por meio de sementes freqüentemente apresenta problemas. Embora existam numerosos registros de consumo de Solanum para morcegos frugívoros (AsCorRa \& WiLson 1992, FLeming 1991, GaletTi \& Morellato 1994, Kunz \& Dias 1995, Mikich 2002, Pedro \& Passos 1995, Sipinski \& Reis 1995, Uieda \& Vasconcellos-Neto 1985, WiLlig et al. 1993), outros gêneros desta família podem ser in-

Revista Brasileira de Zoologia 20 (3): 511-517, setembro 2003 
cluídos na dieta destes animais, como, por exemplo, Aureliana e Vassobia (Tab. III), que possuem sementes muito semelhantes às de Solanum, mesmo quando observadas sob lupa. Faz-se necessária, portanto, uma abordagem cautelosa na análise da dieta de morcegos frugívoros, sendo indispensável a montagem de uma boa coleção de referência de sementes para a identificação em termos de espécies vegetais presentes em sua dieta.

A comunidade de morcegos frugívoros desta área de Mata Atlântica parece explorar eficientemente os recursos disponíveis ao longo do ano, respondendo possivelmente com mudanças na dieta ou deslocamentos para outras áreas de acordo com as variações na oferta de frutos das suas plantas preferenciais ou nas mudanças climáticas. Um maior conhecimento da dinâmica espacial e temporal da interação entre plantas e morcegos frugívoros é fundamental para traçar estratégias de conservação na Mata Atlântica do sudeste brasileiro.

\section{AGRADECIMENTOS}

Este trabalho foi realizado com o apoio do Programa BIOTA/FAPESP - O Instituto Virtual da Biodiversidade (www.biota.org.br), FAPESP (Processos $\mathrm{n}^{\circ} .98 / 05090-6$ e $\mathrm{n}^{\circ} .98 /$ 08940-0) e do CNPq, através da bolsa concedida ao primeiro e terceiro autores (F.C. Passos; W.A. Pedro) e ao último (M.R. Bonin - PIBIC/CNPq). Somos gratos a Burton Lim pela identificação de espécies de morcegos, a Ramoci Leuchtenberger e James J. Roper pelo auxílio em algumas coletas, a Sandro Menezes da Silva, Marilia Borgo e Rodolfo Antônio de Figueiredo pela identificação das exsicatas botânicas, e a um revisor anônimo e Nélio Roberto dos Reis pelas sugestões ao manuscrito. A Fundação Florestal do Estado de São Paulo forneceu o apoio logístico no Parque Estadual Intervales.

\section{REFERÊNCIAS BIBLIOGRÁFICAS}

ArITA, H.T. 1993. Rarity in neotropical bats: correlations with phylogeny, diet, and body mass. Ecological Applications, Ann Arbor, 3 (3): 506-517.

Ascorra, C. F. \& D.E. Wilson. 1992. Bat frugivory and seed dispersal in the amazon, Loreto, Peru. Publicaciones del Museo de Historia Natural Universidad Nacional Mayor de San Marcos (A), Lima, 43: 1-6.

BERNARD, E. 2002. Diet, activity and reproduction of bat species (Mammalia, Chiroptera) in Central Amazonia, Brasil. Revista Brasileira de Zoologia, Curitiba, 19 (1): 173-188.

Charles-Dominique, P. 1986. Inter-relations between frugivorous vertebrates and pioneer plants: Cecropia, birds and bats in French Guyana, p. 119-135. In: A. Estrada \& T.H. Fleming (Eds). Frugivores and seed dispersal. Dordrecht, Dr. W. Junk Publ., 392p.

Cole, F.R. \& D.E. WiLson. 1996. Mammalian diversity and natural history, p. 9-39. In: D.E. Wilson; F.R.ColE; J.D. Nichols; R. RUDRAN \& M.S. Foster (Eds). Measuring and monitoring biological diversity. Standard methods for mammals. Washington, Smithsonian Institution Press, 409.

EMmons, L.H. \& F. FeER. 1997. Neotropical rainforest mammals: a field guide. Chicago, The University of Chicago Press, 392p.

Fenton, M.B.; J.O.Whitaker JR.; M.J. Vonhof; J.M. Waterman; W.A. Pedro; L.M.S. Aguiar; J.E. Baungarten; S. Bouchard; D.M. FaRia; C.V. Portfors; N.I.L. Rautenbach; W. Scully \& M. Zortéa,
1999. The diet of bats from Southeastern Brazil: the relation to echolocation and foraging behaviour. Revista Brasileira de Zoologia, Curitiba, 16 (4): 1081-1085.

FLEMING, T.H. 1986. Opportunism versus specialization: evolution of feeding strategies in frugivorous bats, p. 105-118. In: A. Estrada \& T. H. Fleming (Ed.). Frugivores and seed dispersal. Dordrecht, W. Junk Publisher, XIII+392p.

.1991. The relationship between body size, diet, and habitats use in frugivorous bats, genus Carollia (Phyllostomidae). Journal of Mammalogy, Lawrence, 72 (3): 493-501.

FonsecA, G.A.B. DA. 1985. The vanishing Brazilian Atlantic Forest. Biological Conservation, Paris, 34: 17-34.

Fonseca, G.A.B. DA; A.B. Rylands; C.M.R. Costa; R.B. Machado \& Y.L.R. LeITE. 1994. Livro Vermelho dos Mamíferos Brasileiros Ameaçados de Extinção. Fundação Biodiversitas, Belo Horizonte, 479p.

Galetti, M. \& L.P.C. Morellato. 1994. Diet of the large fruiteating bat Artibeus lituratus in a forest fragment in Brazil. Mammalia, Paris, 58 (4): 661-665.

Gardner, A.L. 1977. Feeding habits, p. 293-350. In: R.J. BAKER; J.K. JONES \& D.C. CARTER (Eds). Biology of bats of the new world family Phyllostomatidae. Lubbock, Special Publication Museum Texas Tech University, \#13, 364p.

Gorchov, D.L.; F. Cornejo; C. Ascorra \& M. Jaramillo. 1993. The role of seed dispersal in the natural regeneration of rain forest after strip-cutting in the Peruvian Amazon, p. 339349. In: T.H. FLEMING \& A. EstRADA (Eds). Frugivory and seed dispersal: ecological and evolutionary aspects. Dordrecht, W. Kluwer Academic Publishers, 416p.

Heithaus, E.R; T.H. Fleming \& P.A. Opler. 1975. Foraging patterns and resource utilization in seven species of bats in a seasonal tropical forest. Ecology, Washington, 56: 841-854.

Kunz, T.H. \& C.A. Diaz. 1995. Folivory in fruit-eating bats, with new evidence from Artibeus jamaicensis (Chiroptera: Phyllostomidae). Biotropica, Lawrence, 27 (1): 106-120.

Lim, B.K.; W.A. Pedro \& F.C. Passos. 2003. Differentiation and species status of the Neotropical yellow-eared bats Vampyressa pusilla and $V$. thyone (Phyllostomidae) with a molecular phylogeny and review of the genus. Acta Chiropterologica, Warsawa, 5 (1): 15-29.

Marinho-FilHo, J.S. 1991. The coexistence of two frugivorous bat species and the phenology of their food plants in Brazil. Journal of Tropical Ecology, Cambridge, 7:59-67.

Мiкich, S.B. 2002. A dieta dos morcegos frugívoros (Mammalia, Chiroptera, Phyllostomidae) de um pequeno remanescente de floresta estacional semidecidual do sul do Brasil. Revista Brasileira de Zoologia, Curitiba, 19 (1): 239-249.

Muller, M.F. \& N.R. ReIs. 1992. Partição de recursos alimentares entre quatro espécies de morcegos frugívoros (Chiroptera, Phyllostomidae). Revista Brasileira de Zoologia, Curitiba, 9 (3/4): 345-355.

NOWAK, R.M \& J.L. Paradiso. 1991. Walker's mammals of the world. Baltimore, Johns Hopkins University Press, vol. 1, $5^{\text {th }}$ ed., 642p.

Pedro, W.A. \& F.C. Passos. 1995. Occurence and food habits of some bat species from the Linhares Forest Reserve, Espirito Santo, Brazil. Bat Research News, New York, 36: 1-2.

Pedro, W.A. \& V.A. Taddei. 1997. Taxonomic assemblage of bats 
from Panga Reserve, Southeastern Brazil: abundance patterns and trophic relations in the Phyllostomidae (Chiroptera). Boletim do Museu de Biologia Mello Leitão, N. Sér., Santa Teresa, 6: 3-21.

. 2002. Temporal distribution of five bat species (Chiroptera, Phyllostomidae) from Panga Reserve, Southeastern Brazil. Revista Brasileira de Zoologia, Curitiba, 19 (3): 951-954.

Pedro, W.A.; C. Carvalho; M.M. Hayashi; A. Bredt; N.M.S. Armani; M.M.S. Silva; L. Gomes; C.A. Gonçalves \& N.F. Peres. 1997. Notes on Vampyressa pusilla (Wagner 1843) in the South of São Paulo State. Chiroptera Neotropical, Brasília, 3 (2): 79-80.

Pedro, W.A.; F.C. Passos \& B.K. Lim. 2001. Morcegos (Chiroptera; Mammalia) da Estação Ecológica dos Caetetus, Estado de São Paulo. Chiroptera Neotropical, Brasília, 7 (1-2): 136-140.

Portfors, C.V.; M.B. Fenton; L.M. De S. Aguiar; J.E. Baugarten; M.J. Vonhof; S. Bouchard; D.M. Faria; W.A. Pedro; N.I.L. Rauntenbach \& M. Zortéa. 2000. Bats from Fazenda Intervales, Southeastern Brazil - species account and comparison between different sampling methods. Revista Brasileira de Zoologia, Curitiba, 17 (2): 533-538.

Reis, N.R. \& J. Guillaumet. 1983. Les chauves-souris frugivores de la région de Manaus et leur rôle dans la dissémination des espèces végétales. Revue D Ecologie - La Terre Et La Vie, Paris, 38: 147-168.

Reis, N.R.; A.L. Peraqui; M.L. Sekiama \& I.P. De Lima. 2000. Diversidade de morcegos (Chiroptera, Mammalia) em fragmentos florestais no estado do Paraná, Brasil. Revista Brasileira de Zoologia, Curitiba, 17 (3): 697-704.

RuI, A.M. \& M.E. Fabián. 1997. Quirópteros de la familia Phyllos- tomidae (Mammalia, Chiroptera) en selvas del estado de Rio Grande do Sul, Brasil. Chiroptera Neotropical, Brasília, 3 (2): 75-77.

SIPINSKI, E.A.B. \& N.R. Reis. 1995. Dados ecológicos dos quirópteros da Reserva Volta Velha, Itapoá, Santa Catarina, Brasil. Revista Brasileira de Zoologia, Curitiba, 12 (3): 519-528.

STONER, K.E. 2001. Differential habitat use and reproductive patterns of frugivorous bats in tropical dry forest of northwestern Costa Rica. Canadian Journal of Zoology, Ottawa, 79: 1626-1633.

Terborgh, J. 1986. Community aspects of frugivory in tropical forests, p. 371-384. In: A. Estrada \& T.H. Fleming (Eds). Frugivores and seed dispersal. Dordrecht, W. Junk Publisher, 392p.

. 1992. Maintenance of diversity in tropical forests. Biotropica, Lawrence, 24: 283-292.

Trajano, E. 1996. Moviments of caves bats in Southeastern Brazil, with emphasis on the population ecology of the common vampire bat, Desmodus rotundus (Chiroptera). Biotropica, Lawrence, 28: 121-129.

Uieda, W. \& J. Vasconcellos-Neto. 1985. Dispersão de Solanum spp. (Solanaceae) por morcegos, na região de Manaus, AM, Brasil. Revista Brasileira de Zoologia, São Paulo, 2: 449-458.

Willig, M.R.; G.R. Camilo \& S.J. Nobile. 1993. Dietary overlap in frugivorous and insectivorous bats from edaphic cerrado habitats of Brazil. Journal of Mammalogy, Lawrence, 74 (1): 117-128.

Zortéa, M. \& A.G. Chiarello. 1994. Observations on the big fruit-eating bat, Artibeus lituratus, in an Urban Reserve of South-east Brazil. Mammalia, Paris, 58 (4): 665-670.

Recebido em 05.XII.2002; aceito em 25.VIII.2003. 